\title{
Cost-Efficient EM-Simulation-Driven Antenna Design Using Co-Kriging
}

\author{
Slawomir Koziel", Stanislav Ogurtsov ${ }^{*}$, Ivo Couckuyt ${ }^{\#}$, and Tom Dhaene ${ }^{\#}$ \\ \#Engineering Optimization \& Modeling Center, School of Science and Engineering, \\ Reykjavik University, Menntavegur 1, 101 Reykjavik, Iceland \\ koziel@ ru.is, stanislav@ ru.is \\ "Surrogate Modeling Lab, Department of Information Technology, \\ Gent University - IBBT, B-9050 Gent, Belgium \\ ivo.couckuyt@ugent.be, tom.dhaene@ugent.be
}

Keywords: Antenna design, electromagnetic simulation, surrogate modeling, Kriging, co-Kriging, computer-aided design.

\begin{abstract}
An efficient and reliable technique for design optimization of antennas is presented. Our approach exploits coarse-discretization electromagnetic (EM) simulations of the antenna of interest that are used to create its fast initial model (a surrogate) through Kriging. During the design process, predictions obtained by optimizing the surrogate are verified using highfidelity EM simulations, and these high-fidelity data are used to enhance the surrogate using co-Kriging interpolation that accommodates all EM simulation data into one surrogate model. The co-Kriging-based optimization algorithm is simple, elegant and is capable of yielding a satisfactory design at a low cost equivalent to a few high-fidelity EM simulations of the antenna under design. To our knowledge, this is a first application of co-Kriging to antenna design. Efficiency of our approach is demonstrated with several antenna design cases and compared to other techniques including pattern search and space mapping.
\end{abstract}

\section{Introduction}

Antenna design is a challenging process that involves adjustment of dimensional and material parameters in order to satisfy various, often conflicting, objectives concerning antenna figures [1]. In many cases, interactions with antenna environment (e.g., housing or connectors) can be influential so they have to be taken into account. Due to this, as well as 
because of the lack of reliable closed form models for many antenna structures (e.g., DRAs [2]), electromagnetic (EM)-simulation-driven design may be the only option.

The major bottleneck of EM-driven design is its high computational cost. Highfidelity antenna simulation may take a few hours so that straightforward approaches employing the EM solver directly in an optimization loop are impractical. In practice, the simulation-driven design process is often carried out through repetitive parameter sweeps where the selection of parameters (usually one at a time) and their ranges are guided by expert knowledge and experience of the designer. Such "tuning" process may take as much as a few weeks of work and involve numerous EM simulations. The EM-driven design cost can be reduced by exploiting adjoint sensitivity techniques [3], [4] that allow obtaining exact derivative information of the antenna responses with respect to the designable parameters at little or no extra computational cost [5]. Recently, adjoint sensitivity has become commercially available (e.g., [6]). The first optimization algorithm exploiting this technology has been implemented in the recent version of the CST Microwave Studio [6]. Still, even with the aid of cheap derivative information, gradient-based optimization may be relatively expensive. Additional problems are due to the fact that EM-based objective functions are usually noisy.

Computationally efficient design can also be realized using surrogate models, fast and yet reasonably accurate representations of the structure of interest. According to this paradigm [7]-[9], direct optimization of the expensive high-fidelity model is replaced by iterative optimization and updating of the surrogate. Under certain circumstances (e.g., when the surrogate model is fast and has good generalization capabilities, surrogate updates require limited number of references to the high-fidelity model), surrogate-based optimization may result in substantial design time savings when compared to conventional design techniques [9].

The surrogate can be created by approximating high-fidelity EM data using loworder polynomials [10], Kriging [11], neural networks [12], [13], support vector regression [14], [15], or rational approximation [16], etc. However, obtaining an accurate model requires dense sampling of the design space (hundreds or thousands of sampled may be necessary), which makes sense for multiple-use library models but not so much for ad-hoc antenna optimization. 
Techniques exploiting physics-based surrogates such as space mapping (SM) [17], [18] and tuning [19], [20] are much more computationally efficient because they construct the surrogate using an underlying low-fidelity (or coarse) model that embeds some knowledge about the physical system under consideration. Unfortunately, applicability of these techniques for antenna design is limited. SM relies on a fast coarse model, typically, circuit equivalent [17]. Reliable circuit models are not available for many important types of antenna. On the other hand, tuning is not directly applicable for radiating structures. Other surrogate-based techniques such as recently introduced shapepreserving response prediction (SPRP) [21] or adaptively adjusted design specifications (AADS) [22] are not as limited as space mapping in terms of the required low-fidelity model speed. The reason is that both SPRP and AADS are parameter-less so that there is no need to extract surrogate model parameters in a nonlinear minimization procedure that is common to SM. On the other hand, neither SPRP nor AADS have been fully automated: some sort of initial user intervention and visual inspection of the model responses is necessary in order to define so-called characteristic points (SPRP) or to modify the design specifications (AADS).

Here, for the first time, we consider application of co-Kriging [23] for antenna design. Co-Kriging allows us to create the surrogate using mostly coarse-discretization EM simulations (which are much cheaper than the high-fidelity ones) and a limited amount of high-fidelity EM data that is accumulated during the iterative process of optimizing and improving the surrogate. Co-Kriging is a natural way to blend EM data of different fidelity, and that allows us to yield an optimized design at a low cost corresponding to a few high-fidelity antenna simulations. Operation and performance of our technique is demonstrated using several antenna design cases. We also compare its efficiency with conventional optimization using pattern search as well as surrogate-based optimization using space mapping.

\section{Antenna Design Using Co-Kriging}

In this section, we formulate the antenna design task as a nonlinear minimization problem, where certain geometry and/or material parameters are to be adjusted so that the structure under consideration meets given performance requirements. We discuss properties 
of low-fidelity antenna models obtained from coarse-discretization EM simulations, as well as described the design optimization procedure. In this procedure the response surface approximation model, initially constructed using solely low-fidelity model data, is gradually improved using the high-fidelity simulations accumulated during the optimization run, and blended with the initial response surface using co-Kriging. The complete optimization algorithm is formulated. Its numerical verification presented in Section 3.

\subsection{Formulation of Antenna Design Problem}

The task of antenna design can be formulated as a following nonlinear minimization problem

$$
\boldsymbol{x}_{f}^{*} \in \arg \min _{\boldsymbol{x}} U\left(\boldsymbol{R}_{f}(\boldsymbol{x})\right)
$$

where $\boldsymbol{R}_{f} \in R^{m}$ denotes the response vector of a high-fidelity (or fine) model of the antenna of interest evaluated through high-fidelity EM simulation; $\boldsymbol{x} \in R^{n}$ is a vector of designable variables, e.g., geometry or material parameters to be adjusted. The response $\boldsymbol{R}_{f}(\boldsymbol{x})$ might be, e.g., the modulus of the reflection coefficient $\left|S_{11}\right|$ evaluated at $m$ different frequencies. $U$ is a given scalar merit function that encodes the design specifications. It is formulated so that a

better design corresponds to a smaller value of $U . \boldsymbol{x}_{f}{ }^{*}$ is the optimal design to be determined. It is assumed that $\boldsymbol{R}_{f}$ is evaluated through computationally expensive EM simulation so that solving (1) directly may be impractical.

\subsection{Low-Fidelity Electromagnetic Models of Antennas}

The design optimization procedure considered in this paper exploits an auxiliary lowfidelity antenna model $\boldsymbol{R}_{c d}$ that is evaluated using the same EM solver as the one used for the high-fidelity model, however, with coarser discretization. In practice, the low-fidelity model can be created not only by reducing the mesh density but also by exploiting one or more of the following options: (i) using smaller computational domain with the finite-volume discrete methods, (ii) using low order basis functions with the finite-element and moment methods, (iii) using staircase shape approximation, (iv) applying simpler absorbing boundary conditions, (v) exciting the computational domain with discrete sources rather than with fullwave ports, (vi) modeling metals with the perfect electric conductors, (vii) neglecting metallization thickness of traces, strips, and patches, or (viii) ignoring dielectric losses and dispersion of materials. 
The main purpose of coarser meshes and other simplifications is to reduce the computational cost of the model. In practice, the time evaluation ratio of the high- and lowfidelity model can be anything between 5 to 50 or more assuming that the accuracy of $\boldsymbol{R}_{c d}$ is reasonable. Higher ratios are possible for certain types of antennas, e.g., for narrow-band and travelling wave antennas. For multi-resonant structures it might not be possible for $\boldsymbol{R}_{c d}$ to adequately represent all the relevant features of the high-fidelity model if the mesh density is not sufficient, particularly at higher frequencies.

A reduced time of model evaluation obviously comes at the expense of degraded accuracy. Therefore, the low-fidelity model cannot directly replace the high-fidelity model in the design problem (1). Nevertheless, it can be used to yield a rough initial approximation of the optimum design. Here, we demonstrate how the low- and high-fidelity EM simulations can be combined using a co-Kriging surrogate in order to substantially speed up the antenna design process.

\subsection{Kriging Interpolation}

A well-known technique in surrogate modeling is Kriging [23], [24], [27]. Kriging surrogate models are also known as Gaussian Processes (GP) [25] or Gaussian Random Fields [26]. For a full discussion of Kriging the reader is referred to [24] and [25]. This section highlights the most important formulae.

Let $X_{B . c}=\left\{\boldsymbol{x}_{c}{ }^{1}, \boldsymbol{x}_{c}{ }^{2}, \ldots, \boldsymbol{x}_{c}^{N . c}\right\}$ be the base (training) set and $\boldsymbol{R}_{c d}\left(X_{B . c}\right)$ the associated low-fidelity model responses. Subsequently, the Kriging interpolant is derived as,

$$
\boldsymbol{R}_{s . K R}(\boldsymbol{x})=\alpha+r(\boldsymbol{x}) \cdot \Psi^{-1} \cdot\left(\boldsymbol{R}_{c d}\left(X_{B . c}\right)-\mathbf{1} \alpha\right)
$$

where 1 is an $N_{c} \times 1$ vector of ones and the coefficient $\alpha$ is determined by generalized least squares,

$$
\alpha=\left(X_{B . c}{ }^{\prime} \Psi^{-1} X_{B . c}\right)^{-1} X_{B . c}{ }^{\prime} \Psi^{-1} \boldsymbol{R}_{c d}\left(X_{B . c}\right) .
$$

$r(\boldsymbol{x})=\left(\psi\left(\boldsymbol{x}, \boldsymbol{x}_{c}^{1}\right), \ldots, \psi\left(\boldsymbol{x}, \boldsymbol{x}_{c}^{N_{c}}\right)\right)$ is an $1 \times N_{c}$ vector of correlations between the point $\boldsymbol{x}$ and the base set $X_{B . c}$, and $\Psi$ is a $N_{c} \times N_{c}$ correlation matrix given by,

$$
\Psi=\left(\begin{array}{ccc}
\psi\left(\boldsymbol{x}_{c}^{1}, \boldsymbol{x}_{c}^{1}\right) & \ldots & \psi\left(\boldsymbol{x}_{c}^{1}, \boldsymbol{x}_{c}^{N_{c}}\right) \\
\vdots & \ddots & \vdots \\
\psi\left(\boldsymbol{x}_{c}^{N_{c}}, \boldsymbol{x}_{c}^{1}\right) & \ldots & \psi\left(\boldsymbol{x}_{c}^{N_{c}}, \boldsymbol{x}_{c}^{N_{c}}\right)
\end{array}\right)
$$

Arguably, the choice of correlation function $\psi(\cdot, \cdot)$ is crucial to create an accurate Kriging surrogate model. A popular class of stationary correlation functions is defined by 
$\psi\left(\boldsymbol{x}, \boldsymbol{x}^{\prime}\right)=\exp \left(\sum_{k=1}^{n}-\theta_{k}\left|x_{k}-x^{\prime}{ }_{k}\right|^{p}\right)$. The smaller the distance between two points, the higher the correlation and, hence, the more the prediction of one point is influenced by the other. Similarly, if the distance increases the correlation drops to zero. The rate and manner at which this happens is governed by several parameters. In essence, the parameter $p$ determines the 'smoothness' of the prediction. A value of $p=2$ leads to a smooth prediction, but also has strict smoothness requirements on the response $\boldsymbol{R}_{c d}$. With a smaller value of $p$ the correlation decreases much faster as the two points move farther from each other, which is suitable for more sharp (discontinuous) responses. The second set of parameters, $\theta_{1}, \ldots, \theta_{n}$, describes the influence sphere of a point on nearby points for each dimension. This is useful as it describes the linearity of the response and, hence, can be used to identify relevant variables.

Usually, $p$ is set fixed while the parameters $\theta_{1}, \ldots, \theta_{n}$ are identified using Maximum Likelihood Estimation (MLE) [29]. In particular, we minimize the negative concentrated lnlikelihood,

$$
\ln (\mathcal{L}) \cong-\frac{N_{c}}{2} \ln \left(\hat{\sigma}^{2}\right)-\frac{1}{2} \ln (|\Psi|)
$$

where,

$$
\hat{\sigma}^{2}=\frac{1}{N_{c}}\left(\boldsymbol{R}_{c d}\left(X_{B . c}\right)-\mathbf{1} \alpha\right)^{\prime} \Psi^{-1}\left(\boldsymbol{R}_{c d}\left(X_{B . c}\right)-\mathbf{1} \alpha\right) .
$$

Often, the parameter $p$ is set to two (= Gaussian correlation function) which is suitable for many engineering problems. However, the problems discussed in this paper have a rather sharp response and, thus, it is chosen to use $p=1$ (= exponential correlation function).

\subsection{Co-Kriging Interpolation}

Co-Kriging is an extension to Kriging, that exploits the correlation between fine and coarse model data to enhance the prediction accuracy [29]. In this paper the autoregressive co-Kriging model of Kennedy et al. [28] is adopted, which, in essence, assumes the fine model is always correct. Hence, a co-Kriging model always interpolates the fine model data, while the coarse model data act as weights, influencing the interpolation in regions where fine model data is absent.

Creating a co-Kriging model can be interpreted as constructing two Kriging models in a sequence. First a Kriging model $\boldsymbol{R}_{s . K R c}$ of the coarse data $\left(X_{B . c}, \boldsymbol{R}_{c}\left(X_{B . c}\right)\right)$ is constructed. 
Subsequently, the second Kriging model $\boldsymbol{R}_{S . K R d}$ is constructed on the residuals $\left(X_{B . f}, \boldsymbol{R}_{d}\right)$, where $\boldsymbol{R}_{d}=\boldsymbol{R}_{f}\left(X_{B . f}\right)-\rho \cdot \boldsymbol{R}_{S . K R c}\left(X_{B . f}\right)$ (the parameter $\rho$ is included in the MLE)

Note that the configuration, e.g.,the choice of the correlation function, of both Kriging models can be adjusted separately for the coarse data $R_{f}$ and the residuals $R_{d}$, respectively.

Finally, the co-Kriging model $\boldsymbol{R}_{S}$ is built upon the two Kriging models. Namely, the co-Kriging interpolant is defined similarly as (1),

$$
\boldsymbol{R}_{s}(\boldsymbol{x})=M \alpha+r(\boldsymbol{x}) \cdot \Psi^{-1} \cdot\left(\boldsymbol{R}_{d}-F \alpha\right)
$$

where the block matrices $M, F, r(\boldsymbol{x})$ and $\Psi$ can be written as a function of the two underlying Kriging models $\boldsymbol{R}_{s . K R c}$ and $\boldsymbol{R}_{s . K R d \text { : }}$

$$
\begin{gathered}
r(\boldsymbol{x})=\left[\rho \cdot \sigma_{c}^{2} \cdot r_{c}(\boldsymbol{x}), \rho^{2} \cdot \sigma_{c}^{2} \cdot r_{c}\left(\boldsymbol{x}, X_{B . K R_{f}}\right)+\sigma_{d}^{2} \cdot r_{d}(\boldsymbol{x})\right] \\
\Psi=\left[\begin{array}{cc}
\sigma_{c}^{2} \Psi_{c} & \rho \cdot \sigma_{c}^{2} \cdot \Psi_{c}\left(X_{B . K R_{c}}, X_{B . K R_{f}}\right) \\
0 & \rho^{2} \cdot \sigma_{c}^{2} \cdot \Psi_{c}\left(X_{B . K R_{f}}, X_{B . K R_{f}}\right)+\sigma_{d}^{2} \cdot \Psi_{d}
\end{array}\right] \\
F=\left[\begin{array}{cc}
\mathbf{1} & \mathbf{0} \\
\rho \cdot \mathbf{1} & \mathbf{1}
\end{array}\right], M=\left[\begin{array}{ll}
\rho & 1
\end{array}\right]
\end{gathered}
$$

where $\left(\sigma_{c}, \Psi_{c}\right)$ and $\left(\sigma_{d}, \Psi_{d}\right)$ are matrices obtained from the Kriging models $\boldsymbol{R}_{s . K R c}$ and $\boldsymbol{R}_{\text {s.KRd}}$, respectively (see Section 2.3). In particular, $\sigma_{c}^{2}$ and $\sigma_{d}^{2}$ are process variances, while $\Psi_{c}(\cdot, \cdot)$ and $\Psi_{d}(\cdot, \cdot)$ denote correlation matrices of two datasets with the optimized $\theta_{1}, \ldots, \theta_{n}$ parameters and correlation function of the Kriging models $\boldsymbol{R}_{s . K R c}$ and $\boldsymbol{R}_{\text {s.KRd }}$, respectively. Similarly to Section 2.3 , we choose the exponential correlation function for the Kriging models, $\boldsymbol{R}_{s . K R c}$ and $\boldsymbol{R}_{s . K R d}$.

Note that the initial construction of a co-Kriging model requires two separate likelihood optimizations, one for each underlying Kriging model. Depending on the number of samples this might be relatively expensive, e.g., the number of low-fidelity samples in particular may be large. However, in this work subsequent updates to the coKriging model only involves new high-fidelity data (the low-fidelity data remains unchanged) and, hence, only the second Kriging model $\boldsymbol{R}_{S . K R d}$ needs to be updated each iteration, which can be done very fast.

\subsection{Design Optimization Procedure Using Co-Kriging}

The proposed design optimization procedure can be summarized as follows. 
1. Set the initial design $\boldsymbol{x}^{\text {init }}$; Optimize $\boldsymbol{R}_{c}$ to find $\boldsymbol{x}^{(0)}$ - initial design for the co-Kriging optimization;

2. Sample $\boldsymbol{R}_{c}$ in the vicinity of $\boldsymbol{x}^{(0)}$ to obtain $\left(X_{B . c}, \boldsymbol{R}_{c}\left(X_{B . c}\right)\right)$;

3. Set $i=0$;

4. Evaluate $\boldsymbol{R}_{f}$ at $\boldsymbol{x}^{(i)}$; Create a co-Kriging model $\boldsymbol{R}_{s}^{(i)}$ as in (2) using $\left(X_{B . c}, \boldsymbol{R}_{c}\left(X_{B . c}\right)\right)$ and $\left(X_{B . f}, \boldsymbol{R}_{f}\left(X_{B . f}\right)\right)$ with $X_{B . f}=\left\{\boldsymbol{x}^{(0)}, \ldots, \boldsymbol{x}^{(i)}\right\}$;

5. Find $\boldsymbol{x}^{(i+1)}$ by optimizing $\boldsymbol{R}_{s}^{(i)}$; Set $i=i+1$;

6. If $\left\|\boldsymbol{x}^{(i)}-\boldsymbol{x}^{(i-1)}\right\|<\varepsilon$ (here, $\varepsilon=10^{-2}$ ) terminate, else go to 5;

Note that the co-Kriging model is created in the vicinity of the $\boldsymbol{R}_{c}$ optimum, which is the best approximation of the optimal design we can get at a low cost. This allows us to use a limited number of $\boldsymbol{R}_{c}$ samples while creating the surrogate. The size of the vicinity is typically 5 to 20 percent of the design space. The training points of $X_{B . c}$ are allocated using Latin Hypercube Sampling [30]. The initial co-Kriging surrogate is created using only one evaluation of $\boldsymbol{R}_{f}$ and then updated using the designs obtained by optimizing the surrogate. The co-Kriging model is implemented as in [32]. By definition $\boldsymbol{R}_{s}^{(i)}\left(\boldsymbol{x}^{(k)}\right)=\boldsymbol{R}_{f}\left(\boldsymbol{x}^{(k)}\right)$ for $k=$ $0, \ldots, i$, so that the surrogate accuracy constantly improves in the vicinity of the expected optimum upon the algorithm convergence. For all test cases considered here (cf. Section 3), this property was sufficient for the algorithm convergence. The algorithm can be optionally embedded in the trust region framework [31] to alleviate possible convergence problems.

\section{Verification Examples}

In this section, a numerical verification of the co-Kriging-based antenna optimization procedure is provided. We consider three examples of antenna structures, a planar monopole, a dielectric resonator antenna, and a wideband slot antenna.

\subsection{Wideband Monopole Antenna}

Consider a monopole antenna shown in Fig. 1. The design variables are $\boldsymbol{x}=\left[l_{1} l_{2} l_{3}\right.$ $\left.w_{1}\right]^{T}$. The input microstrip of the monopole is fed through an edge mount SMA connector. Simulation time of the coarse-discretization model $\boldsymbol{R}_{c}(223,728$ mesh cells) is $3 \mathrm{~min}$, and that of $\boldsymbol{R}_{f}(1,733,892$ mesh cells) is 60 min. Both models are evaluated using the transient solver of CST Microwave Studio [6]. The design specifications for reflection are $\left|S_{11}\right| \leq-15 \mathrm{~dB}$ for 3 $\mathrm{GHz}$ to $6 \mathrm{GHz}$. The initial design is $\boldsymbol{x}^{\text {init }}=\left[\begin{array}{llll}2 & 2 & 2 & 0\end{array}\right]^{T} \mathrm{~mm}$. The antenna was optimized using 
the co-Kriging-based algorithm of Section 2.5. The approximate optimum of $\boldsymbol{R}_{c}, \boldsymbol{x}^{(0)}=[22.1$ $3.440 .019 .1]^{T}$, is obtained at the cost of 80 evaluations of $\boldsymbol{R}_{c}$. The co-Kriging surrogate is created in the region $\left[\boldsymbol{x}^{(0)}-\boldsymbol{\delta}, \boldsymbol{x}^{(0)}+\boldsymbol{\delta}\right.$, with $\boldsymbol{\delta}=\left[\begin{array}{llll}1 & 0.5 & 0.5 & 1\end{array}\right]^{T}$, using $50 \boldsymbol{R}_{c}$ samples.

The co-Kriging optimization process is accomplished in 5 iterations with the optimized design $\boldsymbol{x}^{(5)}=\left[\begin{array}{lll}21.56 & 3.08-0.09920 .11\end{array}\right]^{T}\left(\left|S_{11}\right|<-16.2 \mathrm{~dB}\right.$ for 3 to $\left.6 \mathrm{GHz}\right)$. Figure 2 shows the reflection responses of $\boldsymbol{R}_{c}$ and $\boldsymbol{R}_{f}$ at $\boldsymbol{x}^{\text {init }}, \boldsymbol{x}^{(0)}$ and $\boldsymbol{x}^{(5)}$. Figure 3 shows the radiation response at the final design $x^{(5)}$. The total design cost (Table 1) corresponds to only 11 evaluations of $\boldsymbol{R}_{f}$. For the sake of comparison, the antenna was also designed through direct optimization of the high-fidelity model using pattern search [33] as well as surrogate-based optimization using space mapping (SM) [17]. SM algorithm is an iterative procedure of the form $\boldsymbol{x}^{(i+1)}=\operatorname{argmin}\left\{\boldsymbol{x}: U\left(\boldsymbol{R}_{s}^{(i)}(\boldsymbol{x})\right\}\right.$, where $\boldsymbol{x}(i), i=0,1, \ldots$, are approximate solutions to the original problem (1). Here, we use output SM surrogate $\boldsymbol{R}_{s}^{(i)}(\boldsymbol{x})=\boldsymbol{R}_{c}(\boldsymbol{x})+\left[\boldsymbol{R}_{f}\left(\boldsymbol{x}^{(i)}\right)-\boldsymbol{R}_{c}\left(\boldsymbol{x}^{(i)}\right)\right]$ that ensures zero-order consistency, i.e., $\boldsymbol{R}_{f}\left(\boldsymbol{x}^{(i)}\right)=\boldsymbol{R}_{c}\left(\boldsymbol{x}^{(i)}\right)$, and does not require parameter extraction [17], which is important from the point of view or reducing the computational overhead of the design process (the low-fidelity model is an EM-simulation one and parameter extraction would require multiple evaluations of $\boldsymbol{R}_{c}$ ). Pattern search converges after 153 evaluations of $\boldsymbol{R}_{f}$ (final design [21.44 2.94-0.142 19.72] $]^{T},\left|S_{11}\right|<-16.4 \mathrm{~dB}$ for 3 to 6 $\mathrm{GHz}$ ). Output SM needs 8 iterations with the total cost of 25 equivalent $\boldsymbol{R}_{f}$ evaluations (final design $\left[\begin{array}{lll}21.513 .57-0.05620 .67\end{array}\right]^{T},\left|S_{11}\right|<-16.3 \mathrm{~dB}$ for 3 to $6 \mathrm{GHz}$ ). All of the designs are comparable, but the computational cost of SM and pattern search is higher. The number of SM iterations is similar to that for co-Kriging, however, SM suffers from extra overhead related to multiple evaluations of the low-fidelity model while optimizing the SM surrogate.

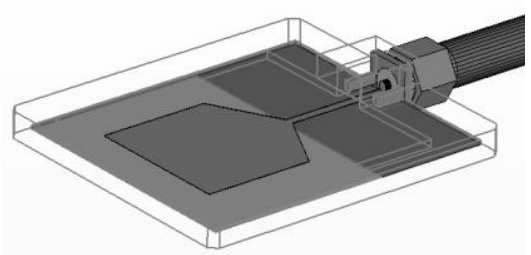

(a)

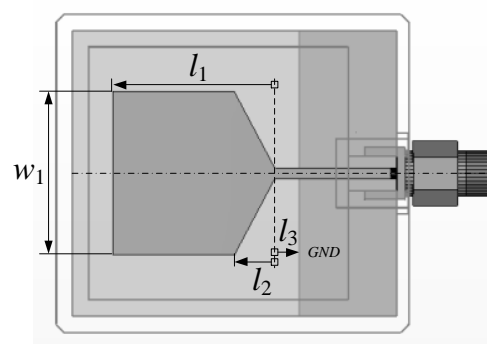

(b)

Fig. 1. Wideband monopole: (a) 3D view; (b) top view. The housing is shown transparent. 


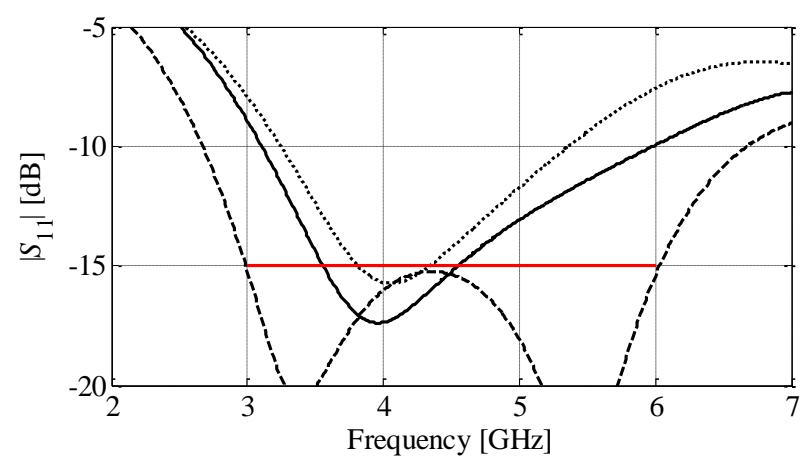

(a)

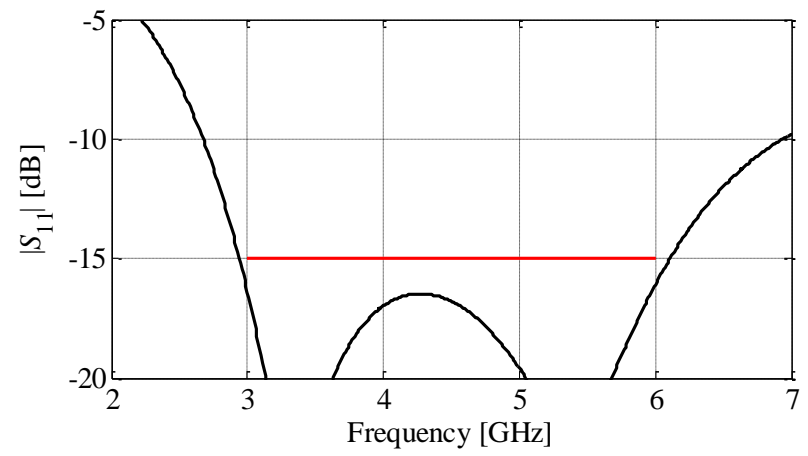

(b)

Fig. 2. Wideband monopole: (a) responses of the low-fidelity model at $\boldsymbol{x}^{\text {init }}(\cdots)$ and $\left.\boldsymbol{x}^{(0)}(--)^{-}\right)$and the response of the high-fidelity model at $\boldsymbol{x}^{\text {init }}(-)$; (b) response of $\boldsymbol{R}_{f}$ at the final design (-).

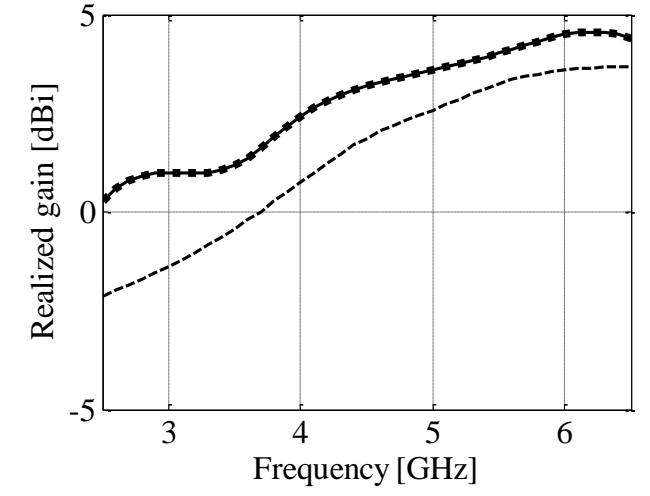

(a)

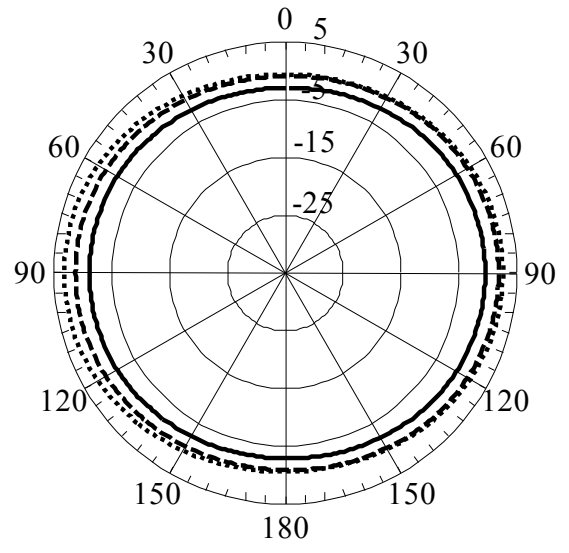

(b)

Fig. 3. Radiation response of the wideband monopole: (a) total realized peak gain (-), theta-pol. realized peak gain (- •), and theta-pol. broadside realized peak gain (- -); (b) theta-pol. broadside realized gain pattern $[\mathrm{dBi}]$ at $3.5(-), 4.5(--)$, and $5.5(\cdot) \mathrm{GHz}$. 
Table 1. Wideband Monopole: Design Cost

\begin{tabular}{ccccc}
\hline \hline \multirow{2}{*}{ Algorithm } & \multirow{2}{*}{$\begin{array}{c}\text { Algorithm } \\
\text { Component }\end{array}$} & $\begin{array}{c}\text { Number of Model } \\
\text { Evaluations }\end{array}$ & \multicolumn{2}{c}{ CPU Time } \\
\cline { 5 - 5 } & Evaluation of $\boldsymbol{R}_{c}{ }^{1}$ & $130 \times \boldsymbol{R}_{c}$ & $390 \mathrm{~min}$ & 6.5 \\
This work (Co- & Absolute & Relative to $\boldsymbol{R}_{f}$ \\
$\begin{array}{c}\text { Kriging-based } \\
\text { optimization) }\end{array}$ & Evaluation of $\boldsymbol{R}_{f}{ }^{2}$ & $5 \times \boldsymbol{R}_{f}$ & $300 \mathrm{~min}$ & 5.0 \\
\hline Pattern search [33] & Total cost & N/A & $690 \mathrm{~min}$ & $\mathbf{1 1 . 5}$ \\
\hline \multirow{2}{*}{ Output SM [17] } & Evaluation of $\boldsymbol{R}_{f}$ & $153 \times \boldsymbol{R}_{f}$ & $300 \mathrm{~min}$ & $\mathbf{1 5 3 . 0}$ \\
& Evaluation of $\boldsymbol{R}_{f}{ }^{2}$ & $335 \times \boldsymbol{R}_{f}$ & $1005 \mathrm{~min}$ & 16.7 \\
& Total cost & $8 \times \boldsymbol{R}_{f}$ & $480 \mathrm{~min}$ & 8.0 \\
\hline \hline
\end{tabular}

80 evaluations to optimize $\boldsymbol{R}_{c}$ and 50 evaluations to set up the co-Kriging model.

${ }^{2}$ Excludes evaluation of $\boldsymbol{R}_{f}$ at the initial design.

${ }^{3}$ Total number of $\boldsymbol{R}_{c}$ evaluations necessary to optimize the SM surrogate.

\subsection{Dielectric Resonator Antenna (DRA)}

Consider a suspended rectangular DRA in a housing shown in Fig. 4. The DRA comprises: a rectangular dielectric resonator with the core permittivity and loss tangent values of 10 and $10^{-4}$, respectively; two Teflon suspension slabs; a polymer housing with the permittivity and loss tangent values of 2.8 and $10^{-2}$, respectively; a ground plane of infinite lateral extends with the feeding slot in it; a $50 \mathrm{~mm}$ thick RO4003 substrate with a $50 \mathrm{ohm}$ input microstrip terminated by an open end section.

The design variables are $\boldsymbol{x}=\left[\begin{array}{llllll}a_{x} & a_{y} & a_{c} & u_{s} & w_{s} & y_{s} \\ g_{1}\end{array}\right]^{T}$. The high-fidelity model $\boldsymbol{R}_{f}$ is simulated using the CST MWS transient solver [6] (763,840 mesh cells at the initial design, evaluation time $20 \mathrm{~min}$ ). The design objective is to obtain $\left|S_{11}\right| \leq-15 \mathrm{~dB}$ for 5.1 $\mathrm{GHz}$ to $5.9 \mathrm{GHz}$. Requirements imposed on the IEEE gain in the frequency band 5.1 $\mathrm{GHz}$ to $5.9 \mathrm{GHz}$ are: minimum gain $5 \mathrm{dBi}$ at the zero zenith angle, and the back-tofront gain less than $-10 \mathrm{~dB}$. Gain requirements are implemented as the design

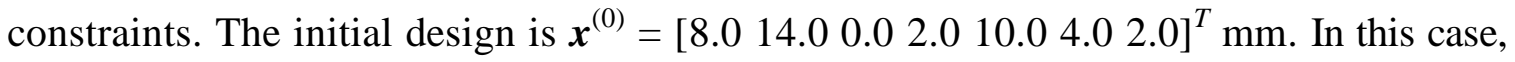
we again exploit the low-fidelity model $\boldsymbol{R}_{c d}$, also evaluated in CST (30,720 mesh cells at $\boldsymbol{x}^{(0)}$, evaluation time 45 seconds).

The antenna was optimized using the co-Kriging-based algorithm starting from the

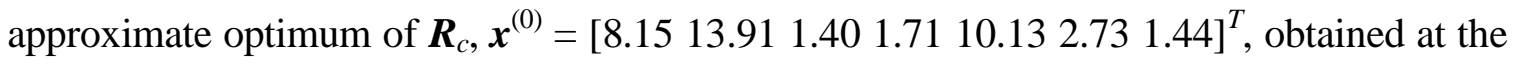
cost of 100 evaluations of $\boldsymbol{R}_{c}$. The co-Kriging surrogate is created in the region $\left[\boldsymbol{x}^{(0)}-\boldsymbol{\delta}, \boldsymbol{x}^{(0)}\right.$ 


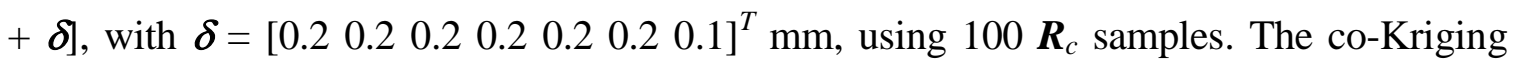
optimization process is accomplished in 6 iterations with the optimized design $\boldsymbol{x}^{(6)}=[8.33$ $13.801 .641 .8110 .082 .741 .59]^{T} \mathrm{~mm}\left(\left|S_{11}\right|<-16.2 \mathrm{~dB}\right.$ for 5.1 to $\left.5.9 \mathrm{GHz}\right)$. Figure 5 shows the responses of $\boldsymbol{R}_{c}$ and $\boldsymbol{R}_{f}$ at $\boldsymbol{x}^{\text {init }}, \boldsymbol{x}^{(0)}$ and $\boldsymbol{x}^{(6)}$. The total design cost corresponds to less than 13 evaluations of $\boldsymbol{R}_{f}$ (Table 2). Similarly to section 3.1, the antenna was also designed through direct optimization of the high-fidelity model using pattern search [33] as well as surrogate-based optimization using space mapping (SM) [17] (the same setup as for the previous example). Pattern search converges after 151 evaluations of $\boldsymbol{R}_{f}$ (final design [8.2

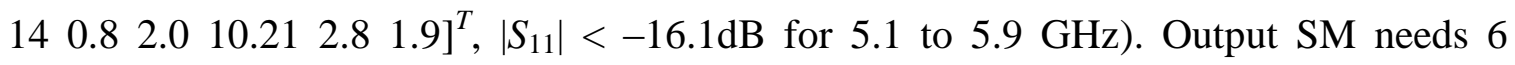
iterations with the total cost of 21 equivalent $\boldsymbol{R}_{f}$ evaluations (final design [8.24 13.9 1.58 $1.6410 .472 .731 .73]^{T},\left|S_{11}\right|<-16.4 \mathrm{~dB}$ for 5.1 to $5.9 \mathrm{GHz}$ ). While all the designs are comparable, the computational cost of both space mapping and pattern search is higher than that of co-Kriging.

\subsection{Broadband Slot Antenna}

Consider a coplanar waveguide (CPW)-fed slot antenna shown in Fig. 6. The design variables are $\boldsymbol{x}=\left[a_{x} a_{y} a b\right]^{T} ; w_{0}=4 \mathrm{~mm}, s_{0}=0.3 \mathrm{~mm}, s_{1}=1.7 \mathrm{~mm}$. The substrate, $0.813 \mathrm{~mm}$ Rogers RO4003C $\left(\varepsilon_{1}=3.38\right.$ at $\left.10 \mathrm{GHz}\right)$, and the ground plane are of infinite lateral extends. The high-fidelity model $\boldsymbol{R}_{f}$ is evaluated with the CST MWS transient solver [6] ( 3,000,000 mesh cells, simulated in $40 \mathrm{~min}$ ). the low-fidelity model is also evaluated in CST MWS ( 45,000 mesh cells, simulation time 50 seconds). The initial design is $\boldsymbol{x}^{(0)}=\left[\begin{array}{lll}40 & 30 & 10\end{array}\right.$ 20 $^{T}$ $\mathrm{mm}$. The design specifications are $\left|S_{11}\right| \leq-10 \mathrm{~dB}$ for 2.3-to-7.6 GHz.

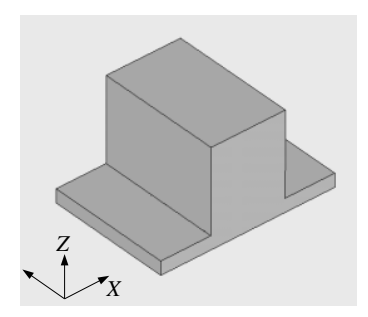

(a)

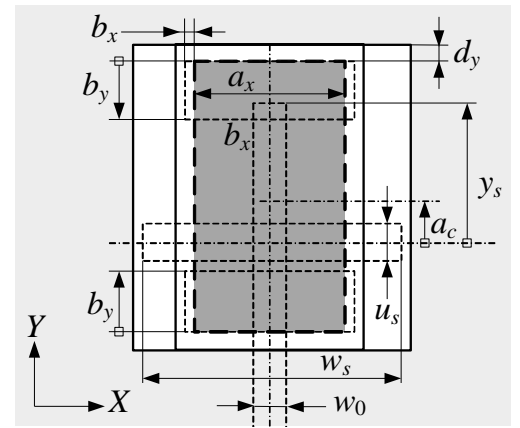

(b)

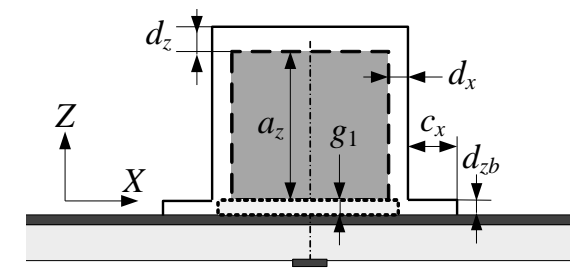

(c)

Fig. 4. DRA: (a) 3D view of its housing, top (b) and front (c) views. 


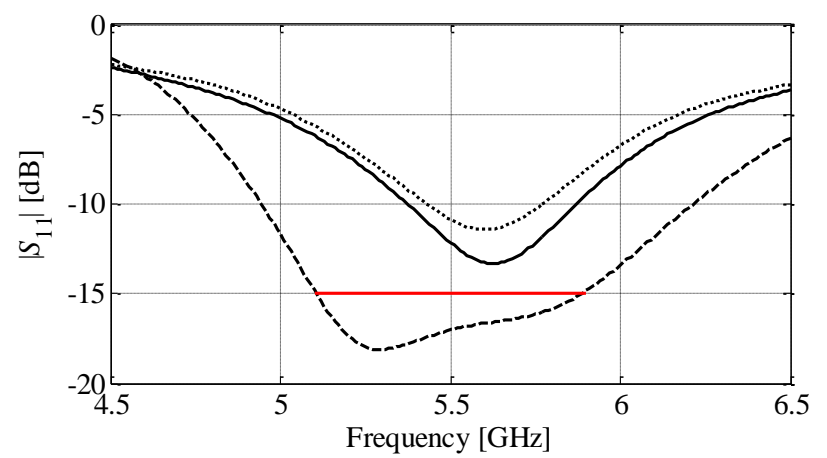

(a)

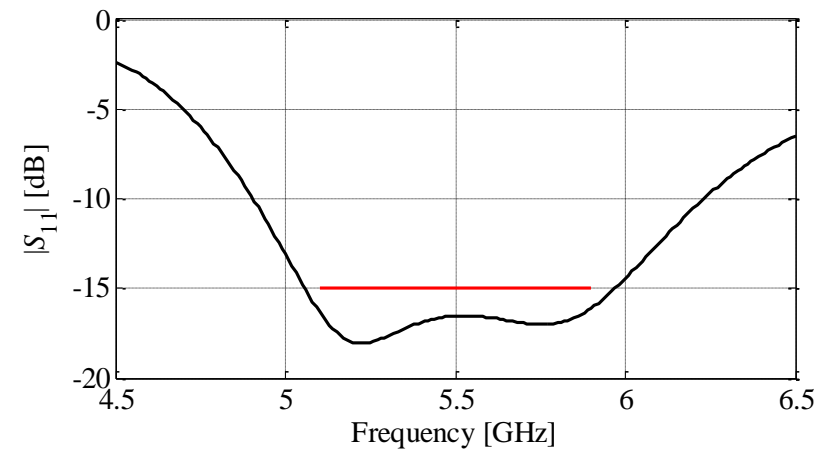

(b)

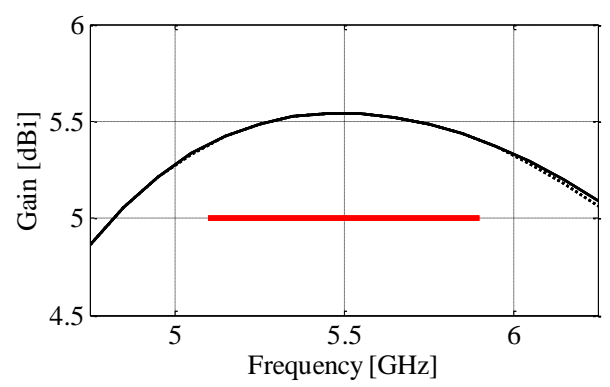

(c)

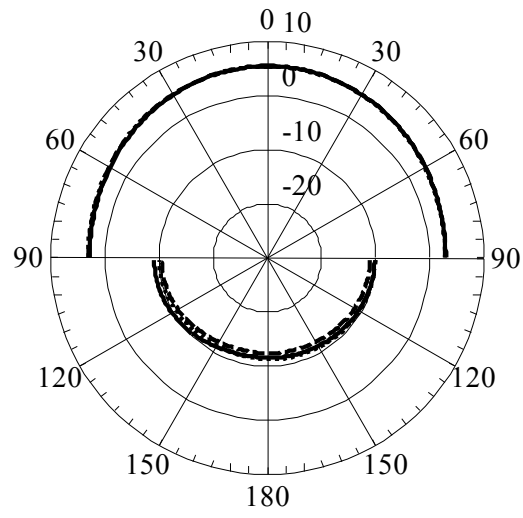

(e)

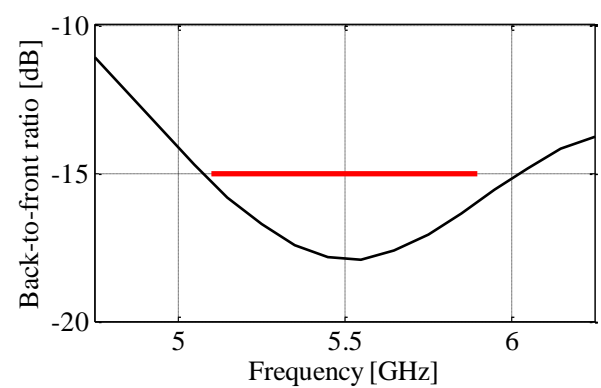

(d)

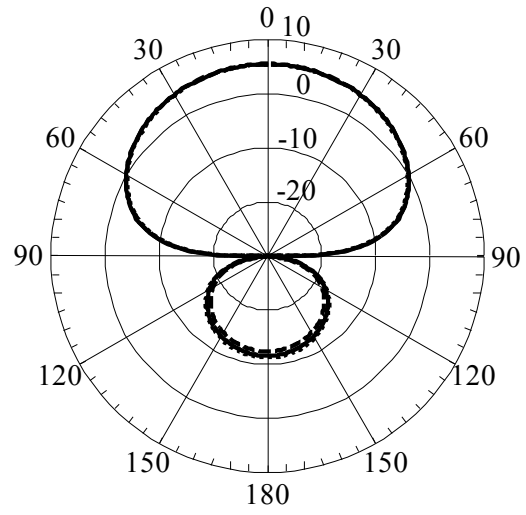

(f)

Fig. 5. DRA: (a) reflection responses of the low-fidelity model at $\boldsymbol{x}^{\text {init }}(\ldots)$ and $\boldsymbol{x}^{(0)}(---)$ and the response of the high-fidelity model at $\boldsymbol{x}^{\text {init }}(-)$; (b) reflection response of the high-fidelity model at the final design (-); (c) peak gain (-) and gain for the zero zenith angle ( $\cdots$ ); (d) back-to-front ratio; (e) gain pattern (co-pol.) in the E-plane at 5.25(-), $5.5(--)$, and 5.75 (*) GHz; (f) gain pattern (cross-pol.) in the H-plane plane at 5.25(-), $5.5(--)$, and $5.75(\cdots) \mathrm{GHz}$. 
Table 2. DRA: Design Cost

\begin{tabular}{ccccc}
\hline \hline \multirow{2}{*}{ Algorithm } & \multirow{2}{*}{$\begin{array}{c}\text { Algorithm } \\
\text { Component }\end{array}$} & $\begin{array}{c}\text { Number of Model } \\
\text { Evaluations }\end{array}$ & \multicolumn{2}{c}{ CPU Time } \\
\cline { 5 - 5 } & Evaluation of $\boldsymbol{R}_{c}{ }^{1}$ & $180 \times \boldsymbol{R}_{c}$ & $135 \mathrm{~min}$ & 6.7 \\
This work (Co- & Absolute & Relative to $\boldsymbol{R}_{f}$ \\
$\begin{array}{c}\text { Kriging-based } \\
\text { optimization) }\end{array}$ & Evaluation of $\boldsymbol{R}_{f}{ }^{2}$ & $6 \times \boldsymbol{R}_{f}$ & $120 \mathrm{~min}$ & 6.0 \\
\hline Pattern search [33] & Total cost & N/A & $255 \mathrm{~min}$ & $\mathbf{1 2 . 7}$ \\
\hline \multirow{2}{*}{ Output SM [17] } & Evaluation of $\boldsymbol{R}_{f}$ & $151 \times \boldsymbol{R}_{f}$ & $300 \mathrm{~min}$ & $\mathbf{1 5 3 . 0}$ \\
& Evaluation of $\boldsymbol{R}_{f}{ }^{2}$ & $400 \times \boldsymbol{R}_{f}$ & $300 \mathrm{~min}$ & 15.0 \\
& Total cost & $6 \times \boldsymbol{R}_{f}$ & $120 \mathrm{~min}$ & 6.0 \\
\hline \hline
\end{tabular}

$\overline{1} 100$ evaluations to optimize $\boldsymbol{R}_{c}$ and 100 evaluations to set up the co-Kriging model.

${ }^{2}$ Excludes evaluation of $\boldsymbol{R}_{f}$ at the initial design.

${ }^{3}$ Total number of $\boldsymbol{R}_{c}$ evaluations necessary to optimize the SM surrogate.

The approximate optimum of the low-fidelity model $\boldsymbol{R}_{c}, \boldsymbol{x}^{(0)}=\left[\begin{array}{lll}43.5 & 28.98 .92\end{array}\right.$ $22.5]^{T} \mathrm{~mm}$, is obtained at the cost of 100 evaluations of $\boldsymbol{R}_{c}$. The antenna was subsequently optimized using the co-Kriging-based algorithm of Section 2.5. The coKriging surrogate is created in the region $\left[x^{(0)}-\delta, x^{(0)}+\delta\right.$, with $\delta=\left[\begin{array}{lllll}2.0 & 2.0 & 1.0 & 1.0\end{array}\right]^{T}$, using $80 \boldsymbol{R}_{c}$ samples. The co-Kriging optimization process is accomplished in 5 iterations with the optimized design $\boldsymbol{x}^{(5)}=\left[\begin{array}{llll}41.51 & 27.25 & 8.91 & 22.15\end{array}\right]^{T}\left(\left|S_{11}\right|<-12.0 \mathrm{~dB}\right.$ for 2.3 to 7.6 GHz) The reflection responses of the low- and high-fidelity model at $\boldsymbol{x}^{\text {init }}, \boldsymbol{x}^{(0)}$ and $\boldsymbol{x}^{(5)}$ are shown in Fig. 7. The overall design cost (Table 3) corresponds to just above 9 evaluations of $\boldsymbol{R}_{f}$. Similarly as for the previous examples, the antenna was also designed through pattern search [33] optimization and through space mapping (SM) optimization [17] (the same setup as for the previous examples). Pattern search converges after 160 evaluations of $\boldsymbol{R}_{f}$ (final design $\left[\begin{array}{llll}41.51 & 27.24 & 8.91 & 22.15\end{array}\right]^{T},\left|S_{11}\right|<-11.8 \mathrm{~dB}$ for 2.3 to 7.6 $\mathrm{GHz}$ ). Output SM needs 7 iterations with the total cost of about 16 equivalent $\boldsymbol{R}_{f}$ evaluations (final design $\left[\begin{array}{lll}37.82 & 23.47 & 9.2022 .60\end{array}\right]^{T},\left|S_{11}\right|<-11.3 \mathrm{~dB}$ for 2.3 to $7.6 \mathrm{GHz}$ ). The computational cost of both space mapping and pattern search is higher than the cost of co-Kriging-based algorithm, whereas the design quality is comparable for all cases. 


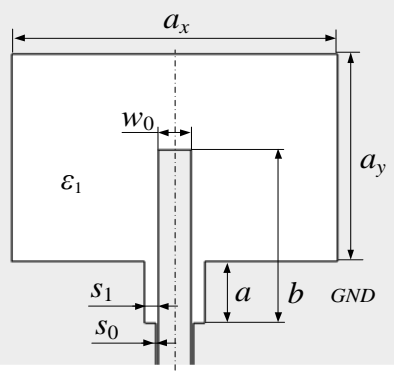

Fig. 6. Geometry of CPW-fed broadband slot antenna.

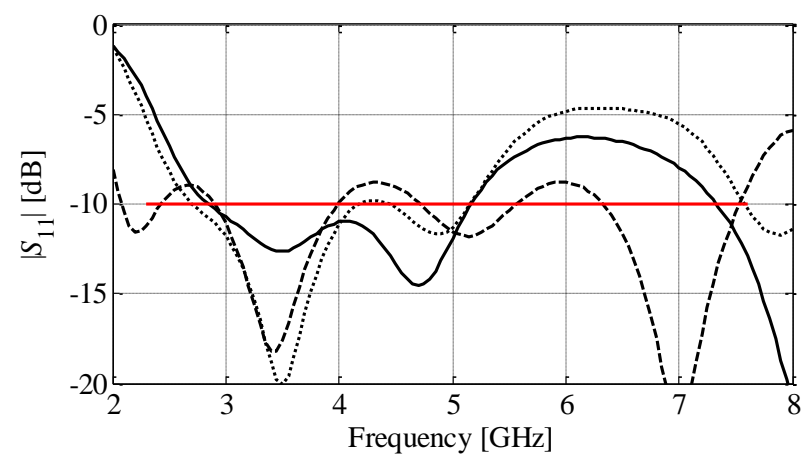

(a)

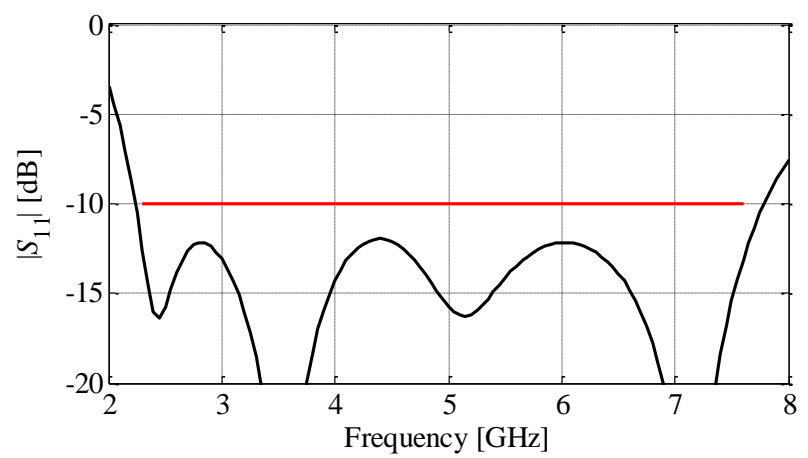

(b)

Fig. 7. CPW-fed broadband slot antenna: (a) reflection responses of the low-fidelity model at $\boldsymbol{x}^{\text {init }}$ $(\cdots)$ and $x^{(0)}\left(-\right.$ - $^{-}$) and the response of the high-fidelity model at $\boldsymbol{x}^{\text {init }}$ (-); (b) response of the high-fidelity model at the final design (-).

\section{Conclusion}

A computationally efficient and reliable procedure for design optimization of antenna structure has been presented. The main component of the procedure is the coKriging interpolation technique that combines the low-fidelity EM simulation data (used to create the initial response surface antenna model) and the high-fidelity EM data accumulated during the optimization process. The comprehensive numerical verification conducted using three examples of antenna structures indicate that the proposed procedure is robust and the results are consistent for all considered test cases. 
Table 3. CPW-Fed Broadband Slot Antenna: Design Cost

\begin{tabular}{ccccc}
\hline \hline \multirow{2}{*}{ Algorithm } & \multirow{2}{*}{$\begin{array}{c}\text { Algorithm } \\
\text { Component }\end{array}$} & $\begin{array}{c}\text { Number of Model } \\
\text { Evaluations }\end{array}$ & \multicolumn{2}{c}{ CPU Time } \\
\cline { 4 - 5 } & Evaluation of $\boldsymbol{R}_{c}{ }^{1}$ & $200 \times \boldsymbol{R}_{c}$ & $167 \mathrm{~min}$ & 4.2 \\
This work (Co- & Absolute & Relative to $\boldsymbol{R}_{f}$ \\
Kriging-based & Evaluation of $\boldsymbol{R}_{f}{ }^{2}$ & $5 \times \boldsymbol{R}_{f}$ & $200 \mathrm{~min}$ & 5.0 \\
\hline Pattern search [36] & Evaluation of $\boldsymbol{R}_{f}$ & $160 \times \boldsymbol{R}_{f}$ & $300 \mathrm{~min}$ & $\mathbf{1 5 3 . 0}$ \\
\hline & Evaluation of $\boldsymbol{R}_{c}{ }^{3}$ & $450 \times \boldsymbol{R}_{f}$ & $375 \mathrm{~min}$ & 9.4 \\
Output SM [17] & Evaluation of $\boldsymbol{R}_{f}{ }^{2}$ & $7 \times \boldsymbol{R}_{f}$ & $280 \mathrm{~min}$ & 7.0 \\
& Total cost & N/A & $655 \mathrm{~min}$ & $\mathbf{1 6 . 4}$ \\
\hline \hline
\end{tabular}

$\overline{1} 100$ evaluations to optimize $\boldsymbol{R}_{c}$ and 100 evaluations to set up the co-Kriging model.

${ }^{2}$ Excludes evaluation of $\boldsymbol{R}_{f}$ at the initial design.

${ }^{3}$ Total number of $\boldsymbol{R}_{c}$ evaluations necessary to optimize the SM surrogate.

\section{Acknowledgement}

This work was supported in part by the Icelandic Centre for Research (RANNIS) Grant 110034021. Ivo Couckuyt is funded by the Institute for the Promotion of Innovation through Science and Technology in Flanders (IWT-Vlaanderen). The authors would like to thank Computer Simulation Technology AG, Darmstadt, Germany, for making CST Microwave Studio available.

\section{References}

[1] J.L. Volakis (Ed.), Antenna engineering handbook, McGraw-Hill, 2007.

[2] A. Petosa, Dielectric resonator antenna handbook, Artech House, 2007.

[3] J.I. Toivanen, J. Rahola, R.A.E. Makinen, S. Jarvenpaa, and P. Yla-Oijala, "Gradient-based antenna shape optimization using spline curves," Annual Review of Progress in Applied Comp. Electromagnetics, Tampere, Finland, pp. 908-913, 2010.

[4] D. Nair and J. P. Webb, "Optimization of microwave devices using 3-D finite elements and the design sensitivity of the frequency response," IEEE Trans. Magn., vol. 39, no. 3, pp. 1325-1328, May 2003.

[5] M.A. El Sabbagh, M.H. Bakr, and N.K. Nikolova, "Sensitivity analysis of the scattering parameters of microwave filters using the adjoint network method," Int. J. RF and Microwave Computer-Aided Eng., vol. 16, no. 6, pp. 596-606, Nov. 2006.

[6] CST Microwave Studio, ver. 2011, CST AG, Bad Nauheimer Str. 19, D-64289 Darmstadt, Germany, 2011.

[7] S. Koziel, D. Echeverría-Ciaurri, and L. Leifsson, "Surrogate-based methods," in S. Koziel and X.S. Yang (Eds.) Computational Optimization, Methods and Algorithms, Series: Studies in Computational Intelligence, Springer-Verlag, pp. 33-60, 2011.

[8] N.V. Queipo, R.T. Haftka, W. Shyy, T. Goel, R. Vaidynathan, and P.K. Tucker, "Surrogate-based analysis and optimization," Progress in Aerospace Sciences, vol. 41, no. 1, pp. 1-28, Jan. 2005. 
[9] Q.S. Cheng, J.W. Bandler, S. Koziel, M.H. Bakr, and S. Ogurtsov, "The state of the art of microwave CAD: EM-based optimization and modeling," Int. J. RF and Microwave Computer-Aided Eng., vol. 20, no. 5, pp. 475-491, 2010.

[10] T.W. Simpson, J. Peplinski, P.N. Koch, and J.K. Allen, "Metamodels for computer-based engineering design: survey and recommendations," Engineering with Computers, vol.17, no. 2, pp. 129-150, July 2001.

[11] A.I.J. Forrester and A.J. Keane, "Recent advances in surrogate-based optimization," Prog. in Aerospace Sciences, vol. 45, no. 1-3, pp. 50-79, Jan.-April, 2009.

[12] J.E. Rayas-Sánchez, "EM-based optimization of microwave circuits using artificial neural networks: the state-of-the-art," IEEE Trans. Microwave Theory Tech., vol. 52, no. 1, pp. 420-435, Jan. 2004.

[13] H. Kabir, Y. Wang, M. Yu, and Q.J. Zhang, "Neural network inverse modeling and applications to microwave filter design," IEEE Trans. Microwave Theory Tech., vol. 56, no. 4, , pp. 867-879, April 2008.

[14] J. Meng and L. Xia, "Support-vector regression model for millimeter wave transition," Int. J. Infrared and Milimeter Waves, vol. 28, no. 5, pp. 413-421, May 2007.

[15] M. Martinez-Ramon and C. Christodoulou, "Support vector machines for antenna array processing and electromagnetics," Synthesis Lectures on Computational Electromagnetics, vol. 1, no. 1, 2006.

[16] G.S.A. Shaker, M.H. Bakr, N. Sangary, and S. Safavi-Naeini, "Accelerated antenna design methodology exploiting parameterized Cauchy models," PIER-99, pp. 279-309, 2009.

[17] J.W. Bandler, Q.S. Cheng, S.A. Dakroury, A.S. Mohamed, M.H. Bakr, K. Madsen, and J. Søndergaard, "Space mapping: the state of the art," IEEE Trans. Microwave Theory Tech., vol. 52, no. 1, pp. 337-361, Jan. 2004.

[18] S. Koziel, Q.S. Cheng, and J.W. Bandler, "Space mapping," IEEE Microwave Magazine, vol. 9, no. 6, pp. 105-122, Dec. 2008.

[19] J.C. Rautio, "EM-component-based design of planar circuits," IEEE Microwave Magazine, vol. 8, no. 4, pp. 79-90, Aug. 2007.

[20] D.G. Swanson and R.J. Wenzel, "Fast analysis and optimization of combline filters using FEM,” IEEE MTT-S IMS Digest, Boston, MA, pp. 1159-1162, July 2001.

[21] S. Koziel, "Shape-preserving response prediction for microwave design optimization," IEEE Trans. Microwave Theory and Tech., vol. 58, no. 11, pp. 2829-2837, 2010.

[22] S. Koziel, "Adaptively adjusted design specifications for efficient optimization of microwave structures," Progress in Electromagnetic Research B (PIER B), vol. 21, pp. 219-234, 2010.

[23] M.C. Kennedy and A. O'Hagan, "Predicting the output from complex computer code when fast approximations are available", Biometrika, vol. 87, pp. 1-13, 2000.

[24] J. Kleijnen, Design and Analysis of Simulation Experiments, Springer, 2008.

[25] C.E. Rasmussen and C.K.I. Williams, "Gaussian Processes for Machine Learning", MIT Press, 2006.

[26] M. T. M. Emmerich, K. Giannakoglou and B. Naujoks, "Single- and multiobjective evolutionary optimization assisted by Gaussian random field metamodels", IEEE Trans. On Evolutionary Computation, vol. 10, pp. 421-439, 2006.

[27] D. Krige, A statistical approach to some basic mine valuation problems on the Witwatersrand, Journal of the chemical, metallurgical and mining society of South Africa, vol. 52, pp. 119-139, 1951.

[28] J. Sacks, W.J. Welch, T. Mitchell and H.P. Wynn, "Design and analysis of computer experiments", Statistical Science, vol. 4, no. 4, pp. 409-435, 1989.

[29] A.I. Forrester, A. Sobester and A.J. Keane, "Multi-fidelity optimization via surrogate modelling", Royal Society, Proceedings of the royal Society, vol. 463, pp. 3251-3269, 2007. 
[30] B. Beachkofski and R. Grandhi, "Improved distributed hypercube sampling," American Institute of Aeronautics and Astronautics, paper AIAA 2002-1274, 2002.

[31] A.R. Conn, N.I.M. Gould, and P.L. Toint, Trust Region Methods, MPS-SIAM Series on Optimization, 2000.

[32] D. Gorissen, I. Couckuyt, P. Demeester, T. Dhaene, and K. Crombecq, "A Surrogate Modeling and Adaptive Sampling Toolbox for Computer Based Design," Journal of Machine Learning Research, vol. 11, pp. 2051-2055, July 2010.

[33] T.G. Kolda, R.M. Lewis, and V. Torczon, "Optimization by direct search: new perspectives on some classical and modern methods," SIAM Rev., 45, pp. 385—482, 2003. 